\title{
The features of surface plasmon resonance in gold cluster films
}

\author{
L.S. Maksimenko, I.E. Matyash, S.P. Rudenko, B.K. Serdega \\ V. Lashkaryov Institute of Semiconductor Physics, NAS of Ukraine \\ 45, prospect Nauky, Kyiv 03028, Ukraine \\ Phone: 38-(044) 525-57-78; e-mail: serdega@isp.kiev.ua
}

\begin{abstract}
The internal reflection of nanosized gold cluster films was studied using the technique of polarization modulation of electromagnetic radiation in the Kretschmann geometry. We measured the reflection coefficients $R_{s}$ and $R_{p}$ of $s$ - and $p$-polarized radiation, respectively, as well as their polarization difference $\Delta R=R_{s}-R_{p}$, as function of the light incidence angle in the $0.4 \div 1.6 \mu \mathrm{m}$ wavelength range. A topological size effect was found; it consists in dependence of the value and sign of curvature of the polarization difference characteristics on the film surface properties. It is shown that the sign of curvature of $\Delta R$ characteristics depends on the radiation wavelength $\lambda$ and indicates resonance interaction with a metal film of either $p$-polarized radiation only or that of both polarizations. The spectral characteristic of the topological size effect in the resonance interaction is obtained from the condition of isotropic reflection, $\Delta R=R_{s}-R_{p}$ $=0$, and its dependence on the radiation wavelength.
\end{abstract}

Keywords: radiation polarization, plasmon-polariton resonance, gold cluster film, topological size effect.

Manuscript received 11.02.09; accepted for publication 18.03.09; published online 20.03.09.

\section{Introduction}

The anomalies of optical properties occur in limited metal objects (films, clusters, nano-shells, wires etc.) whose sizes are comparable with the electron free path. First of all, these anomalies lead to appearance of the classical size effect [1]. The topological size effect [2] related to the form of object surface also occurs in that range of sizes. When both effects occur in the same object of investigation (say, a rough film as a cluster structure and its continuous ultrathin underlayer), then it is difficult to separate the contributions of each of the reasons for those effects.

It is this situation that takes place when studying the surface plasmon-polariton resonance (PPR) that is observed in thin films of some metals (in particular, gold). The thicknesses of samples where PPR occurs most clearly depend on the wavelength of the radiation used and lie within a range of several tens of nanometers [3]. In thinner films where the concept of thickness is represented by a mass of deposited substance, PPR (called the local plasmon resonance [4]) reflects not only the anomalies of dielectric properties of the material under consideration [5] but the features of surface topology as well. In this case, the traditional methods applied for investigation of metal nanolayers (e.g., transmission [6], absorption [7] and luminescence [8]) have limited capabilities for separation of dimensional features in interaction between the electromagnetic field of the wave and electron system. Moreover, the condition of resonance interaction (that requires equality of the value of dispersion for the wave and plasmonpolariton [9] and is strictly obeyed in the films with geometrically flat surface) loses rigor of the orientation dependence in cluster structures. There, in particular, the dielectric function becomes spatially- or sizedependent [10].

Therefore, the objective of this work is, first of all, investigation of the influence of the properties of a cluster structure on the polarization characteristics of the internal reflectance of gold samples. The traditional opinion is that PPR in such samples manifests itself only in the dependence of the reflection coefficient $R_{p}$ of $p$ polarized radiation on the angle of light incidence. It will be shown that the above influence leads also to dependence of the reflection coefficient $R_{S}$ of $s$-polarized radiation and the polarization difference $\Delta R=R_{s}-R_{p}$ on the angle of light incidence.

As to the concept of sample structure as functional argument (in spite of some indeterminacy of that concept), the situation becomes simpler because of some physico-technological reasons. It is well known that, owing to the growth conditions, a thin metal layer on a dielectric substrate is a composite that consists of two components: cluster and continuous uniform ones. Therefore, one might expect that, by exerting control 
over the interrelation between the above components, it would be possible to obtain a result characterizing their separate effects on the quantity measured. In our case, such a quantity is the polarization difference of the coefficients of internal reflection that is registered with the polarization modulation (PM) technique.

Application of the above technique for investigation of PPR in ultrathin gold films is implicit another part of the problem. It seems expedient for the following reason. Both internal and external reflections are characterized with amplitude and phase anisotropies. For our experiment, the most important is the fact that generally the values and angular dependences of the reflection coefficient for $s$-polarized radiation differ from the corresponding characteristics of the reflection coefficient for $p$-polarized radiation. This distinction is known as the polarization difference. Being an experimentally measured quantity, it is a product of the PM technique because it is formed by periodically varying in time radiation with orthogonal polarization azimuths.

The sense of polarization difference is as follows. After subtraction, the common features of the functions of the effect under investigation (optical, photoelectric, magnetooptical etc.) vanish, and the residual contains the individual features of the terms. In many cases, this residual is much below the terms. However, it can be reliably registered with the PM technique owing to amplification. As has been shown earlier [11], application of PM for investigation of the features of total internal reflection attenuated with gold films made it possible to separate the PPR characteristics against the background of two nonresonance components. This fact gives grounds to use the PM technique for investigation of cluster nanostructures as one more demonstrations of its detection abilities.

\section{The samples and experimental procedure}

Our samples were gold ( $\mathrm{Au}-99.999 \%)$ films prepared with thermal evaporation in a vacuum (pressure of $10^{-}$ ${ }^{3} \mathrm{~Pa}$ ) from a molybdenum heater onto a substrate kept at room temperature. Glass plates served as substrates. They contacted with a glass segment through an immersion film (glycerol), and their thickness was such that they formed a semicylinder $4 \mathrm{~cm}$ in diameter. The fixed rate of gold sputtering $(1.0 \mathrm{~nm} / \mathrm{s})$ enabled us to set the required values of film thickness by varying the process duration. More accurate size measurement was made when studying sample topology with an atomic force microscope (AFM) NanoScope IIIa (produced by Digital Instrument, USA). To this end, we used the mode of periodic contact of film surface with a silicon needle (rated needlepoint up to $10 \mathrm{~nm}$ ).

The mass thicknesses $d$ (nm) of the samples under investigation were 5 (I), 10 (II) and 20 (III). The above values were chosen from the following considerations. The samples of type I (samples-I) were freshly prepared island films. An axonometric AFM-topology of the sample surface is presented in Fig. 1a. One can see that the film consists of separate clusters loosely contacting one another. Their shape was close to a cylinder, with diameter of $30 \div 80 \mathrm{~nm}$ and height of $5 \div 25 \mathrm{~nm}$. The height drop over a $1 \times 1 \mu \mathrm{m}$ area was $30.24 \mathrm{~nm}$ and the root-mean-square (RMS) roughness was $4.95 \mathrm{~nm}$.

The samples-II in the initial state were a gold film with continuous surface - see Fig. 1b. It consisted of grains with fuzzy boundaries; the grain diameter (height) was $20 \div 45 \mathrm{~nm}(0.5 \div 2 \mathrm{~nm})$. The film was very smooth, with height drop of $6.78 \mathrm{~nm}$ and RMS roughness of $0.91 \mathrm{~nm}$. To transform that film into a cluster one, we used thermal treatment, by analogy with [12]. The film was heated in air at a temperature of $230^{\circ} \mathrm{C}$. After thermal annealing the film structure (Fig. 1b) differed from that of film-I (Fig. 1a) but quantitatively. The film material aggregated in separate clusters with irregularly shaped bases, increased diameter $(30 \div 50 \mathrm{~nm})$ and heights of $6 \div 30 \mathrm{~nm}$. The roughness characteristics were close to those of the initial film-I: height drop of $35.89 \mathrm{~nm}$ and RMS roughness of $5.69 \mathrm{~nm}$.

The samples-III (whose initial thickness was $20 \mathrm{~nm}$ ) were initially smooth, with RMS roughness of $1.91 \mathrm{~nm}$. After heating at the above temperature $\left(230{ }^{\circ} \mathrm{C}\right)$, both the film appearance and bulk structure have changed. Although the film mass thickness remained the same $(20 \mathrm{~nm})$, the film became more porous owing to material aggregation in clusters of increased size: diameter of $80 \div 180 \mathrm{~nm}$ and height of $18 \div 25 \mathrm{~nm}$.

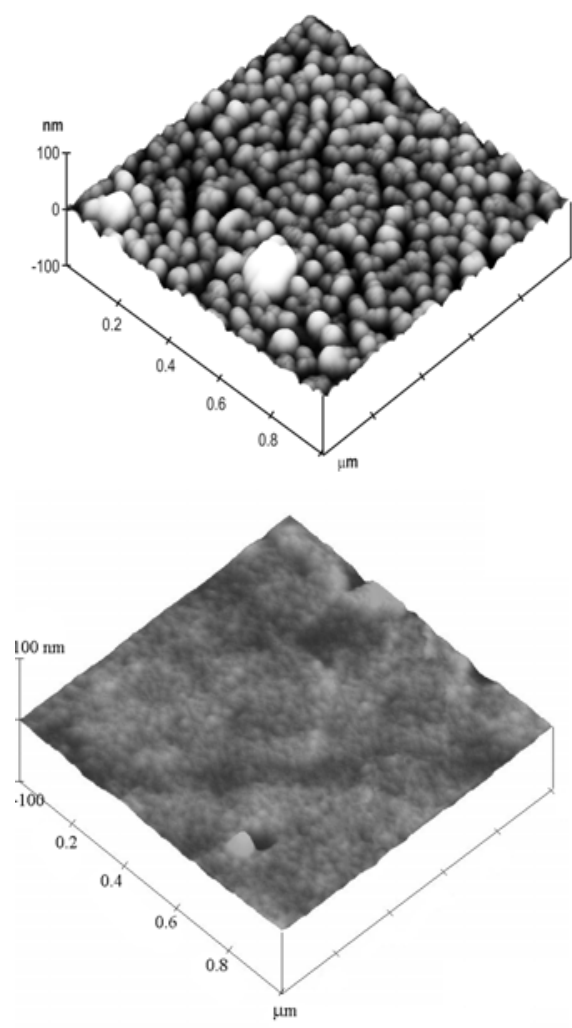

(a)

Fig. 1. AFM-topology of the surfaces of $5 \mathrm{~nm}$ (a) and $10 \mathrm{~nm}(\mathrm{~b})$ gold films. 
The optical unit of the installation for investigation of the PPR features was made in the Kretschmann geometry. (For its diagram as well as detailed description of the processes of modulation of radiation polarization and signal registration, see [11].) Modulation of polarization was made with the most appropriate facility based on the photoelastic effect [13]. The quality of the facility was characterized by the degree of temporal constancy of outgoing radiation intensity; it was $\cong 10^{-4}$ in relative units. The polarization difference $\Delta R=R_{s}-R_{p}$, as well as the separate reflection coefficients $R_{s}$ and $R_{p}$ were measured with the PM technique. The AFM measurements of the films were performed both before and after thermal treatment. The radiation sources were a He-Ne laser (wavelengths of 0.63 and $1.15 \mu \mathrm{m}$ ) and diffraction monochromator with a halogen lamp at the inlet and linear polarizer at the outlet.

\section{Results and discussion}

The polarization characteristics of internal reflection for a sample-I are presented in Fig. 2. The curves 1 and 2 show the reflection coefficients $R_{p}$ and $R_{s}$ as function of the light incidence angle. The curve $1^{\prime}$ (being a typical characteristic of PPR) shows $R_{p}$ dependence obtained for the samples with thickness $d=50 \mathrm{~nm}$ that is optimal for manifestation of the effect.

One can draw the following conclusions from comparison of the curves. First, the extreme values of all the three curves lie at the same angle $\theta_{\text {cr. }}$ Second, the curves have, depending on angle, a typical for PPR dip at $\theta>\theta_{\text {cr }}$. The above two facts enable one to conclude that interaction of electromagnetic radiation with the cluster electron system is of resonance character not only for $p$-polarized radiation but for $s$-polarized radiation as well. As to the distinctions in the values and extents of dips of the $R_{p}$ curves, they are caused exclusively by the cluster structure of the films (see below).

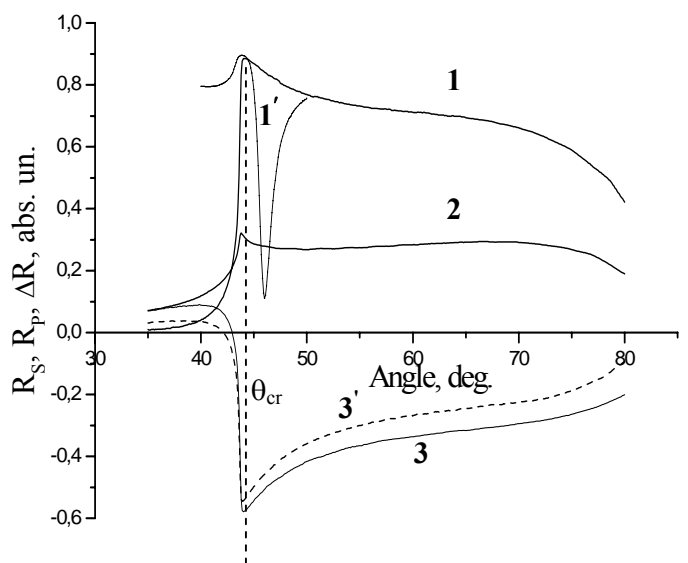

Fig. 2. The dependences of the reflection coefficients $R_{p}\left(1,1^{\prime}\right), R_{s}(2)$ and polarization difference $\Delta R\left(3,3^{\prime}\right)$ on the angle of light incidence for a gold film $\left(l^{\prime}-\right.$ film thickness $d=$ $50 \mathrm{~nm} ; 3^{\prime}-d=10 \mathrm{~nm}$, sample-II after thermal treatment); light wavelength $\lambda=1.15 \mu \mathrm{m}$.
The curve 3 in Fig. 2 presents the polarization difference of reflection coefficients, $\Delta \mathrm{R}$, for a sample-I measured directly with the PM technique. Its negative sign points to the fact that presence of an absorbing medium at the semicylinder surface is the reason for "anomalous" reflection of $s$-polarized radiation in a sense that, contrary to what you might expect, the inequality $R_{s}<R_{p}$ holds near the critical angle. Presence of an almost linear section of $\Delta R$ curve at $\theta>\theta_{\text {cr }}$ reflects the common features of the dependences $R_{s}(\theta)$ and $R_{p}(\theta)$.

The measurements for the sample-II were performed both before and after thermal treatment. The results of the first measurement are presented in Fig. 3a. One can see that there is practically no reflection of the $s$-polarized radiation, and clear attributes of PPR interaction can be seen for the $p$-polarized radiation only. It should be noted that small $R_{p}$ values are observed over a wide range of angles, contrary to the narrow dip shown by curve 1' in Fig. 2. Evidently, this fact is due to film roughness; this is in agreement with somewhat decreased $R_{p}$ value in the maximum near the critical angle.

Thermal treatment has dramatically changed the surface properties of the film as well as its polarization characteristics. First of all, the amplitude of reflection of $p$-polarized radiation near the critical angle increased, while the value of the dip at $\theta>\theta_{\text {cr }}$ decreased (see Fig. 3b, curve 1). The most important result of thermal

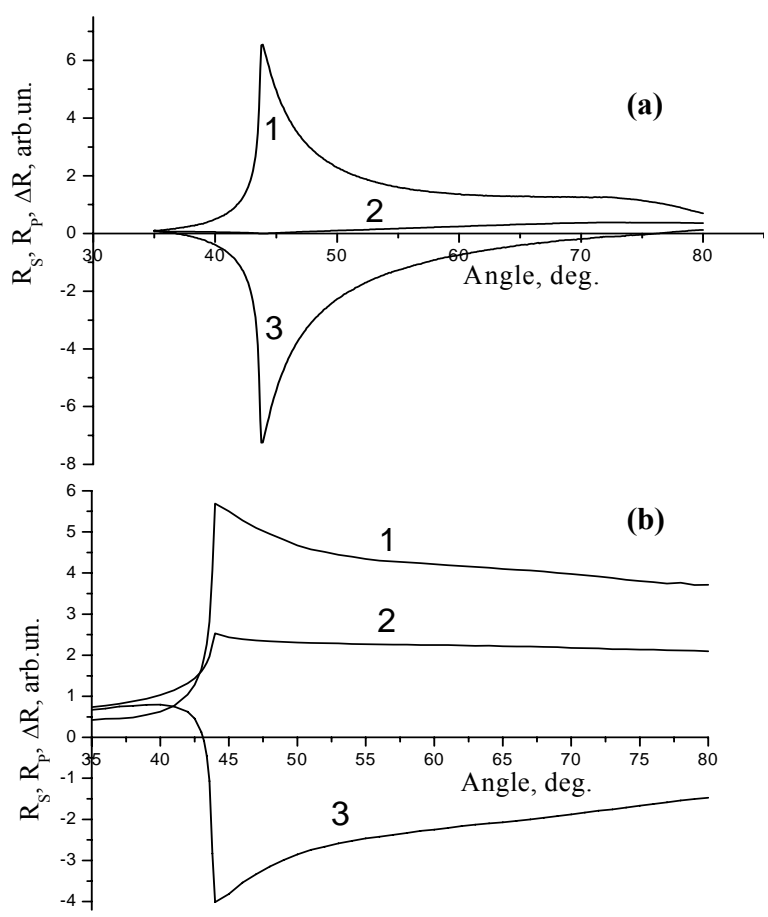

Fig. 3. The dependences of the reflection coefficients $R_{p}(1)$, $R_{s}(2)$ and polarization difference $\Delta R(3)$ on the angle of light incidence for a $10 \mathrm{~nm}$ gold film before (a) and after thermal treatment (b); light wavelength $\lambda=1.15 \mu \mathrm{m}$.

\section{(C) 2009, V. Lashkaryov Institute of Semiconductor Physics, National Academy of Sciences of Ukraine}


treatment was that the reflection coefficient for $s$ polarized radiation grew manifold, the mass thickness of the film remaining the same (curve 2), and the resonance indications appeared in the $R_{s}(\theta)$ curve. The AFMtopologies of the surfaces of the samples with $d=5 \mathrm{~nm}$ (in the initial state - see Fig. 1) and $d=10 \mathrm{~nm}$ (after thermal annealing) are close in both their appearances and parameters. This fact may indicate similarity of the characteristics of the reflection coefficients of the above samples. The experimental result presented in Fig. $3 b$ supports the above assumption. All the three characteristics shown in this Figure practically completely (except some details) coincide with those shown in Fig. 2 for the sample with $d=5 \mathrm{~nm}$. To illustrate, curve $3^{\prime}$ (Fig. 2) that is the polarization difference for the sample with $d=10 \mathrm{~nm}$ after thermal annealing agrees well with similar curve for a thinner sample.

The dipole-dipole, or intercluster, interaction has an impact on the dependence of the form of polarization characteristics on wavelength. This dispersion is pronounced most clearly for the function $\Delta R(\theta)$. They are presented in Fig. 4 for a sample-I (for the sake of clearness and convenience of analysis, they are normalized to the same minimum value). The curve $\lambda=$ $800 \mathrm{~nm}$ stands out of the set of dependences because it is close to straight line over some angle interval. This indicates presence of functionally identical curve pieces in the dependences $R_{s}(\theta)$ and $R_{p}(\theta)$. The experiment shows that the value $\lambda \cong 800 \mathrm{~nm}$ (to which curve 3 corresponds) is limit value in a sense. The formal attribute of the threshold character of that curve is that the characteristics lying on each side of the mentioned one have opposite signs of curvature in the range of angles somewhat above the critical one. What is more important, the value $\lambda \cong 800 \mathrm{~nm}$ for the sample-I is transitional in that resonance character of interaction between radiation and sample is inherent to a greater extent to $p$-polarized radiation of smaller wavelengths, while at bigger wavelength, the resonance character of interaction is inherent to both $s$ - and $p$-polarizations.

It would seem that such an experimental fact is at variance with the condition of PPR excitation according to which only the $p$-polarized wave energy is transferred to a surface plasmon-polariton. However, one could easily imagine a model in which that fact would be explainable based on a spherical (or close to spherical) form of a surface that is characteristic of a cluster film. First, in a cluster of such shape, the electric field vector is oriented at an angle to its surface for both states of wave polarization. Second, by definition of PPR appearance on the spherical surface of a cluster, this becomes possible in a considerable range of angles exceeding the critical one. That is, at any angle (from that range) of light incidence onto the back (working) surface of the semicylinder, there always exists an angle relative to a flat area of cluster surface that obeys the condition of resonance interaction.
All the characteristics of the polarization difference $\Delta R(\theta)$ obtained for the samples under investigation have a paradoxical common feature: at some angles, reflection does not depend on polarization state of the wave. This manifests itself in intersection of the abscissa axis with the $\Delta R(\theta)$ curve within the angle range $0^{\circ}<\theta<90^{\circ}$ and appearance of the equality $R_{s}=R_{p}$ that cannot be described with the Fresnel formulas in what concerns either its position in the angular dependence or especially the number of intersections.

For all the samples studied, there were two intersections of the abscissa axis with the $\Delta R(\theta)$ curve. They lay on each side of the critical angle $\theta_{\text {cr }}$ and indicated isotropic reflection. One of them (laying at $\theta<\theta_{\mathrm{cr}}$ ) was owing to presence of an absorbing medium on the surface of the totally reflecting prism. Its origin is as follows. Action of the electric field of $s$-polarized wave (that is not restricted with the surface) results in bigger absorption of the energy of $s$-polarized wave in the above medium as compared to that of the $p$-polarized wave. The comparative decrease of the reflection coefficient reduction is the reason for "anomalous" reflection.

Another isotropic point in the angular dependence of the polarization difference (laying at $\theta>\theta_{\mathrm{cr}}$ ) was due to resonance interaction of radiation with the electron system of the cluster. Its position on the angle scale depended on to what extent the cluster shape and size met the resonance condition for the $p$-polarized wave (or both polarizations).

This conclusion is in agreement with those attributes of the resonance interaction in the characteristics $R_{s}(\theta)$ and $R_{p}(\theta)$ that are presented in Fig. 2 (curves 1 and 2). If a film is partially cluster, then the angle of isotropic reflection has to be sensitive to the degree of film clusterization because of variation of interrelation between the intensities of differently polarized waves that excite resonance interaction. It is difficult to define the concept of degree of clusterization, at least quantitatively. Therefore, it is more convenient to use the characteristic $\Delta R(\theta)$ of the sample considered for registration of dispersion of isotropic reflection.

Shown in Fig. 4 are the dependences $\Delta R(\theta)$ for a sample-I at different wavelength. One can see that the angle corresponding to the condition of equality of the reflection coefficients depends nonmonotonically on the radiation wavelength. The sample-II after thermal treatment also demonstrates similar dependences with nonmonotonic intersection of the abscissa axis. The characteristic feature of both curves is that the zerovalue point shifts towards total beam grazing due to positive curvature of the functions $\Delta R(\theta)$. In this connection it was of interest to study informativity of the function of isotropic reflection, $\theta_{\Delta R=0}(\lambda)$, with our samples. To this end, we measured the angles $\theta_{\Delta R=0}$ for the samples of all three types, both before and after thermal annealing. The results of our measurements are presented in Fig. 5. Taking into account that, along with 


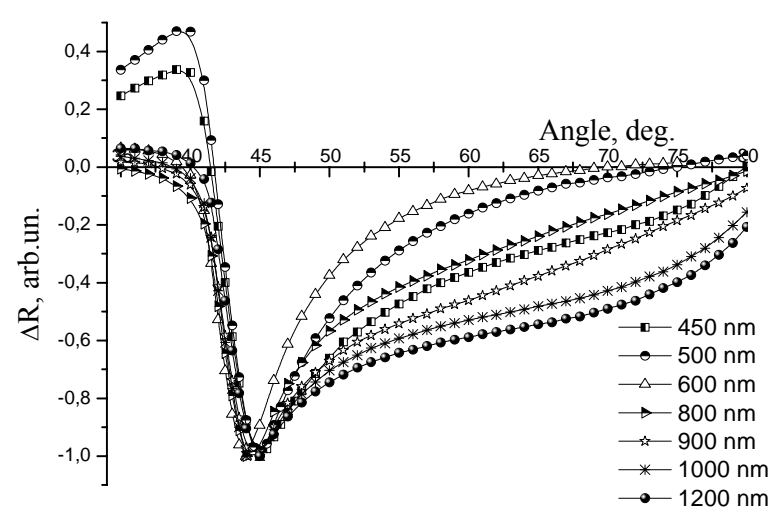

Fig. 4. The dependences of the polarization difference $\Delta R$ on the angle of light incidence for a $5 \mathrm{~nm}$ sample; light wavelength $\lambda=450 ; 500 ; 600 ; 800 ; 900$; and $1200 \mathrm{~nm}$.

the above measurements, we studied also the characteristics $R_{s}(\theta)$ and $R_{p}(\theta)$ to determine interrelation between the intensities of their resonance attributes, interpretation of the presented results does not involve difficulties.

The characteristics shown in Fig. $5 \mathrm{~b}$ as almost horizontal lines at an angle $\theta \cong 85^{\circ}$ (samples-I and samples-II after thermal annealing) indicate possibility to excite PPR with linearly polarized radiation of arbitrary azimuth. The characteristic of the sample-III after thermal treatment demonstrates small changes that seem to be related to variation of the gold film structure. Its position near the angle $\theta \cong 50^{\circ}$ indicates resonance interaction with $p$-polarization interaction only. As to the values of the rest of characteristics at intermediate angles for $\lambda>500 \mathrm{~nm}$, they are determined by the value of contribution to resonance interaction from the $s$ polarized radiation.

This conclusion does not refer to all the $\theta_{\Delta R=0}(\lambda)$ characteristics at wavelengths $\lambda<500 \mathrm{~nm}$. In this case, narrow nonmonotonicity is observed for the samples-I and samples-II. In any event, the nature of the characteristics is not related to PPR. This follows from Fig. 6 presenting the characteristics $\theta_{\Delta R=0}(\lambda)$ for a sample-II in the initial state and after thermal treatment. A comparison of them showed that film restructuring due to heating made an inessential impact on the characteristics of polarization difference in that wavelength range.

The formal reason for the noted features is shift of the isotropic point lying to the left of the critical angle $\theta_{\text {cr. }}$. That point, as was noted, is subjected to influence of the value of extinction coefficient. This is indicated by the known trends in variation of extinction value that are related to film thickness or structural perfection. However, determination of the details of physical origin of such dispersion remains beyond the scope of the present problem.
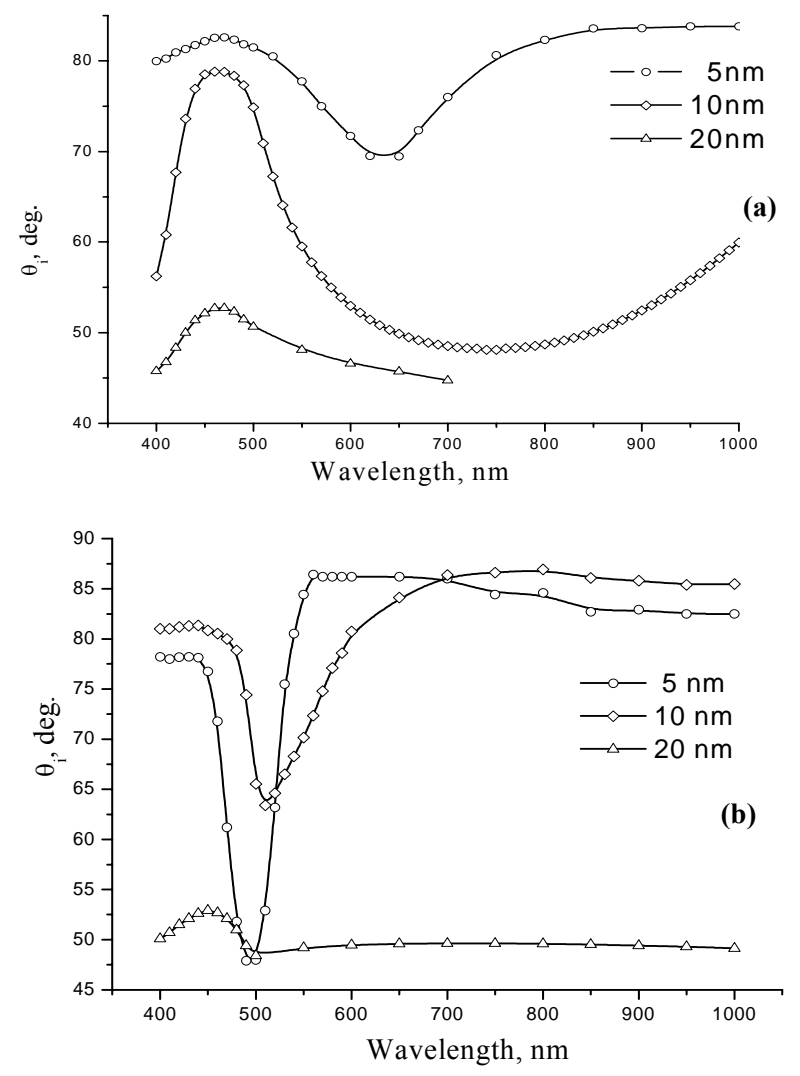

Fig. 5. The spectral dependences of the angle of isotropic reflection for samples 5, 10, and $20 \mathrm{~nm}$ thick before (a) and after thermal treatment (b).

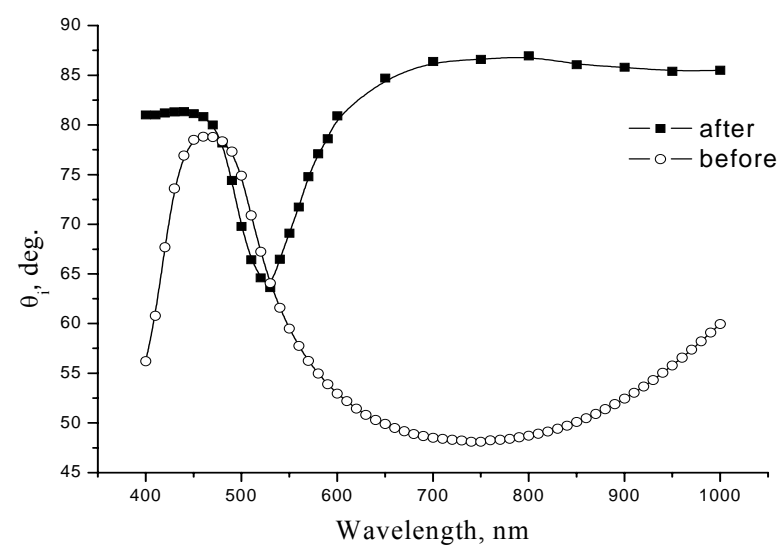

Fig. 6. The spectral dependences of the angle of isotropic reflection for a $10 \mathrm{~nm}$ sample before and after thermal treatment.

The last remark concerns the sample-III for which an inessential effect of film heating was detected by comparing the dependences $\theta_{\Delta R=0}(\lambda)$ obtained before and after thermal annealing. Taking into account the results of AFM studies of the film, one can conclude that the reason for such a small effect was decrease of film roughness owing to material aggregation to bigger bulk clusters. 


\section{Conclusion}

The samples studied in this work demonstrated polarization characteristics of internal reflections with some features that cannot be described with the Fresnel formulas (at least without application of approximations that take into account surface topology or structural properties of the whole film bulk). Nevertheless, the experimental results obtained make it possible to build a system of sample classification according to certain features.

One of them is that the form of $\Delta R(\theta)$ curve dependence on the wavelength that is expressed with the sign and value of function curvature enables one to draw conclusions concerning the degree of film clusterization. This is based on the fact that the resonance mechanism of interaction between radiation and the electron system of clusters takes place not only for $p$-polarized radiation (as it occurs in films that are uniform in thickness) but also for both polarization states (in a certain wavelength range). The reason for such a peculiarity is finite size of clusters and their three-dimensional shape. These factors put restrictions on electron motion under action of the wave field not only along the normal to the film (substrate) surface but also parallel to it that is caused by $s$-polarization.

Another attribute that is more sensitive to the surface properties of a film (and even more to its degree of clusterization) is the characteristic $\theta_{\Delta R=0}(\lambda)$. Its essence is that, at some angle $\theta$ of light incidence, the coefficients of reflection for $s$ - and $p$-polarized radiation become equal and their polarization difference $\Delta R=$ $R_{s}-R_{p}$ vanishes.

The PM technique has resolving power of $0.01^{\circ}$ relative to registration of the angle of isotropic reflection. Measurements of that angle as function of the wavelength of radiation used make it possible to obtain reliable spectral characteristics. From their position in the coordinate space, $\theta_{\Delta R=0}(\lambda)$, conclusions were made concerning (i) interrelation between the intensities of two polarization states at PPR excitation and (ii) cluster character of gold films. It was demonstrated with investigation of gold cluster films that application of the PM procedure for internal reflection, along with the developed interpretation of the characteristics of polarization difference and isotropic reflection, can serve for testing the topological properties of surfaces of thin absorbing films.

\section{References}

1. J. Szczyrbowski, J. Dryzek, A. Czapla, Determination of free-electron parameters of thin films // Thin Solid Films 112, p. 175 (1984).

2. M.L. Dmytruk, O.S. Kondratenko, S.A. Kovalenko, I.B. Mamontova, The classic and topological size effects in optical properties of thin gold films // Fizika Khimiya Tverd. Tila 7(1), p. 39-44 (2006) (in Ukrainian).

3. G. Vuye, Thesis (1981), Univ. P. et M. Curie, Paris VI.

4. E. Hutter, H. Fendler, Exploitation of localized surface plasmon resonance // Adv. Mater. 16(19), p. 1685-1706 (2004).

5. N.L. Dmitruk, V.G. Litovchenko, V.L. Strizhevskii, Surface Polaritons in Semiconductors and Insulators. Naukova Dumka, Kiev, 1989 (in Russian).

6. Jinlian Hu, Woong Lee, Weiping Cai, Liuniu Tong, Haibo Zeng, Evolution of the optical spectra of an $\mathrm{Ag} /$ mesoporous $\mathrm{SiO}_{2}$ nanostructure heat-treated in air and $\mathrm{H}_{2}$ atmosphere // Nanotechnology 18(18), 185710 (2007).

7. Kazuma Kurihara, Carsten Rockstuhl, Takashi Nakano, Tomofumi Arai, Junji Tominaga, The size control of silver nano-particles in $\mathrm{SiO}_{2}$ matrix film // Nanotechnology 16(9), p. 1565-1568 (2005).

8. Byung-hee Choi, Hyun-Ho Lee, Sunmi Jin, Sangki Chun, Sang-Ho Kim, Characterization of the optical properties of silver nanoparticles films // Nanotechnology 18(7), 075706 (2007).

9. Surface Polaritons, Electromagnetic Waves at Surfaces and Interfaces, Eds. V.M. Agranovich, D.L. Mills. North-Holland, Amsterdam and New York, 1982.

10. S.A. Kovalenko, Dimensional effects in thin gold films // Semiconductor Physics, Quantum Electronics \& Optoelectronics 3(4), p. 514-519 (2000).

11. L.I. Berezhinsky, L.S. Maksimenko, I.E. Matyash, S.P. Rudenko, B.K. Serdega, Polarization modulation spectroscopy of surface plasmon resonance // Optika i Spektroskopiya 105(2), p. 281-289 (2008) (in Russian); Optics and Spectroscopy 105(2), p. 257-264 (2008).

12. Makoto Hirai, Ashok Kumar, Wavelength tuning of surface plasmon resonance by annealing silvercopper nanoparticles // J. Appl. Phys. 100, 014309 (2006).

13. S.N. Jasperson, S.E. Schnatterly, An improved method for high reflectivity ellipsometry based on a new polarization modulation technique // Rev. Sci. Instr. 40(6), p. 761-767 (1969). 